ANALYSIS

\title{
In search of social equipoise
}

\begin{abstract}
A failure to acknowledge uncertainty about the effectiveness of social interventions is a major barrier to evidence based public policy making. M Petticrew and colleagues argue that we need to develop and apply the concept of social equipoise
\end{abstract}

\author{
M Petticrew professor, M McKee professor, K Lock senior lecturer, J Green professor, G Phillips \\ research fellow
}

Faculty of Public Health and Policy, London School of Hygiene and Tropical Medicine, London WC1H 9SH, UK

Randomised controlled trials are common in medicine but less so in other fields. However, the use of trials in medicine is helped by an open acknowledgment of uncertainty about effectiveness - that is, clinical equipoise. In theory the same should also apply to social interventions (such as public policies), but no concept of "social equipoise" exists. This makes it politically difficult for policy makers to acknowledge uncertainty and to conduct evaluations. The development of "social equipoise" may help foster a greater culture of evaluation outside medicine. This may be particularly important in England now that public health has moved from the control of primary care trusts to local authorities, which are influenced by party politics.

\section{Equipoise and the role of randomised trials}

The randomised controlled trial is often described as the "gold standard" for evaluating clinical interventions, ${ }^{1}$ and its use has increasingly been advocated in other sectors such as international development and social policy. ${ }^{2}$ Although its roots are in the social sciences rather than medicine, it is rare for it to be used to evaluate innovations in public policy. ${ }^{3}$ For example, the number of randomised trials identified by the Campbell Collaboration, covering education, social welfare, crime, and justice, is a small fraction of the number in the Cochrane Collaboration, with its focus on health. ${ }^{4}$ The reasons for this are the subject of debate but include perceptions that trials are costly and pose practical and ethical difficulties. One recent report for the UK's Cabinet Office challenged several such arguments and included another possible explanation: that policy makers often think that they already know the answer. ${ }^{4}$ However, it did not explicitly consider why they fail to accept that the answer is not known or, in the parlance of clinical trials, that there is equipoise.

Indeed, discussion of equipoise outside clinical trials seems rare, even though it is now widely accepted in clinical research as a key way of determining what Chiong has called "the social merit and therapeutic acceptability" of a clinical trial. ${ }^{5}$ Thus, although previous discussions of the lack of randomised trials in public policy usually focus on practical, ethical, and methodological challenges, the acknowledgment of uncertainty may be just as important a driver to improve the evidence base. Clinical equipoise exists when there is no consensus about the comparative merits of the alternatives to be tested. ${ }^{6}$ Equipoise is a prerequisite for a randomised trial. Miller and Joffe note, "Physicians can ethically randomly assign patients to treatments provided that equipoise - a state of professional uncertainty about their relative therapeutic merits-exists. If equipoise exists, no participant in a randomized clinical trial is knowingly given inferior treatment." Mann also sees the existence of equipoise as creating an imperative to conduct a trial, arguing that "Equipoise motivates clinical experts to propose randomized, controlled trials to resolve uncertainties about the relative merits of interventions."

\section{Reasons for lack of discussion of social equipoise}

In public health policy research the concept of equipoise or even uncertainty may not be salient for several reasons:

Lack of evidence on variations in practice and outcomes-Even a few decades ago the concept of uncertainty was not widely accepted in clinical medicine. Many treatments were adopted on the basis of experience, with no attempt to evaluate them rigorously. This approach was rendered unsustainable by evidence of the extent of variation in practice, which gained prominence with the work of Wennberg and colleagues in the 1980s. ${ }^{9}{ }^{10}$ In contrast, there is much less research documenting variations in public policy and what exists is often between countries, where it is easy to dismiss differences as being due to unspecified national differences. Where variations in public policy are identified, they are often argued to be appropriate responses to variations in need or context.

Multiplicity of outcomes-In complex social interventions there are more likely to be multiple equally important outcomes. This makes the concept of equipoise hard to apply because although 
some stakeholders may agree that there is uncertainty around one outcome, others may feel that there is no real uncertainty around the outcomes they would prioritise. Moreover, "superiority" of one outcome is more difficult to determine and identification of a primary outcome may even be seen as bad research practice because it excludes the values of certain stakeholders. ${ }^{11}$ Thus, urban regeneration programmes, which are often cited as social interventions aimed at improving health, are intended to bring about a range of outcomes, of which health is only one, and not usually the primary one from a policy perspective. ${ }^{12}$ Other outcomes include improvements in the physical environment, employment opportunities, and reductions in crime, and all these may be more important to various stakeholders such as local politicians and the people who live in the area. The outcomes that policy makers value may also depend on their political persuasion-for example, reductions in inequalities may be less important to those on the political right than on the left. ${ }^{13}$

\section{Policy makers or practitioners are unwilling to admit} uncertainty-Even when policy makers believe there is genuine equipoise, they may not be able to admit this publicly for political reasons, particularly when "doing nothing" is not an option. Furthermore, individual values and political beliefs may shape the way that evidence is evaluated and used. In one US study, registered Democrats who were given evidence on the social determinants of diabetes were more likely to support social intervention than those who were not; however, registered Republicans given the same evidence were less likely to support intervention. ${ }^{14}$ Political beliefs can also contribute to confirmation bias, when decision makers seek out evidence consistent with their views and disregard that which contradicts it. $^{15}$

Lack of evidence-The current evidence base makes equipoise difficult to "see." In a field where there is a lot of robust evidence, it may be easier to determine whether you are in equipoise about any particular decision. When the evidence base is very complex, and partial, however, it can be difficult to be clear about whether equipoise exists, or whether equipoise is the appropriate position to adopt—for example, there may be equipoise around the health outcomes but not around the social outcomes.

\section{Difficulties with the concept of equipoise}

The concept of equipoise is not without critics: Miller and Joffe raise several arguments against it as the arbiter of the ethical legitimacy of randomised trials to evaluate new treatments, including imprecision in defining the concept, the reliance on expert opinion to determine when equipoise exists, and the limitations of determining efficacy on the basis of surrogate outcomes. ${ }^{7}$ It may also be particularly difficult to apply in social interventions, where the equipoise is around indirect health effects (such as respiratory health in the case of improvements to social housing) but the primary economic or social effects seem certain. ${ }^{16}$ In this case equipoise may be unlikely and randomisation may even be unethical. ${ }^{17}$

Another difficulty is that the way that problems are constructed in public health and local government is different from the way that they are constructed in clinical settings, so that equipoise does not exist or is not seen as essential. Some local government activities entail responding to local problems with actions whose impact can be easily determined without perfect evidence. The actions they take to improve community safety-for example, changing policing strategies to target antisocial behaviour-may be based on the accumulation of calls, reports, or observations from a geographical location. It is much easier to see in such a situation whether something has "worked" (setting aside the problem of confounding and the counterfactual-whether the situation may have improved anyway or something else might have worked better) if the problem goes away once action is taken. This is very different from, for example, determining whether action taken to reduce rates of coronary heart disease in an area has worked, where the time to outcome is long, and the causal chains are complex and non-linear.

\section{Legitimising uncertainty}

However, aside from these difficulties a strong case can be made for developing a general concept of equipoise in the case of social interventions and policies. This might start by the legitimisation of uncertainty. Uncertainty about a course of action may help foster a culture of evaluation and make it politically easier for randomised trials (as well as other evaluations) of policies to be conducted. In one such example, Camden Council, in London, recognised that there was genuine uncertainty about the effect of a boiler management system aimed at reducing hot water temperatures and heating bills. As well as its effect on heating bills, the council wanted to know whether it would reduce scalds or result in people making more use of kettles to heat water, increasing the risk of spills. To answer these questions a cluster randomised trial in 22 social housing estates was conducted, which showed that scalds were indeed reduced but there was no effect on heating bills. ${ }^{18}$

The argument for a concept of social equipoise was made by the evaluation guru Donald Campbell (though he does not refer to it as equipoise) in his seminal paper "Reforms as Experiments" in $1969 .{ }^{19}$ In it he discusses the difficulty of evaluating the effects of US policy reforms (box). He acknowledges that evaluating outcome is politically risky because it holds the decision maker up to scrutiny and can prove that an intervention supported with public money does not work, at least with respect to the outcomes evaluated. However, Campbell argues that a wider understanding, acknowledgement, and acceptance of the concept of equipoise in public policy would facilitate evaluation and learning and what he calls the "honest evaluation of outcomes."

The move of public health in England to local authorities could facilitate this process. There are many areas within local authority control where there is a need for further evaluation, such as approvals of late night licensing, implementation of 20 mph zones, or funding breakfast clubs for schoolchildren. Such interventions might have a substantial effect on public health and other outcomes ${ }^{20-22}$ but can be controversial. Openness about uncertainty and clarity about the outcomes that are expected would facilitate rigorous evaluation. The transfer of public health staff, who are sensitised to evaluation and trained in its application and interpretation, could encourage this process, but it is too early to know if this will happen. Early indications suggest that the public health capacity and political interest in it at local level may be extremely variable. Research on local government policy making before 1974, when much of the public health function moved into the NHS, suggests that political ideology, and the misplaced sense of certainty that accompanies it, may dominate decision making. ${ }^{23}$ One recent commentator has, for example, pointed to the difference between public health and political timescales: "Public health staff used to thinking in terms of policies which will take many years to demonstrate their effectiveness will need to understand how the three or four-year electoral cycle dominates the thinking of their political bosses." 24 


\section{Reforms as experiments}

"It is one of the characteristics of the present situation that specific reforms are advocated as though they were certain to be successful . . This predicament, abetted by public apathy and by deliberate corruption, may prove in the long run to permanently preclude a truly experimental approach to social amelioration ... by making explicit that a given problem solution was only one of several that an administrator or party could in good conscience advocate, and by having ready a plausible alternative, the administrator could afford honest evaluation of outcomes"

Donald Campbell ${ }^{19}$

Academic debates about the lack of rigorous evidence to support policy making often consider only the methodological arguments. However, fostering a culture of evidence production and use is not simply a matter of disseminating methodology to non-academic colleagues. In this case, developing the concept of "social" equipoise may make it easier for decision makers to conduct new evaluations; may help them think of the conditions in which new trials are needed; and may help reduce what Campbell calls policy makers' "excess of commitment" to particular positive outcomes.

Contributors and sources: The idea for the article emerged from discussions among the authors, who have experience in public health evaluation and developing evidence for public health policy and practice. This article draws on discussions and research with colleagues in local authorities and public health practitioners as part of the work of the NIHR School of Public Health Research (http://sphr.lshtm.ac.uk/). MP, $\mathrm{JG}, \mathrm{MM}$, and GP all have experience in conducting evaluations of policies and local social interventions including housing, transport, and other complex interventions aimed at tackling inequalities. MP wrote the first draft; all authors contributed to subsequent revisions and to addressing reviewers' comments, and approved the final version. MP is the guarantor.

Competing interests: We have read and understood the BMJ Group policy on declaration of interests and have no relevant interests to declare. The authors received funding as part of the NIHR School for Public Health Research. The funder had no role in the paper.

Provenance and peer review: Commissioned; not externally peer reviewed.

1 Evidence Based Medicine Working Group. Evidence-based medicine. A new approach to teaching the practice of medicine. JAMA 1992;268:2420-5.

2 Banerjee A, Duflo E. Poor economics: a radical rethinking of the way to fight global poverty. Public Affairs, 2011

3 Oakley A. Experiments in knowing: gender and method in the social sciences. UK Polity Press, 2000.

4 Haynes L, Service O, Goldacre B, Torgerson D. Test, learn, adapt: developing public policy with randomised controlled trials. Cabinet Office, 2012.
5 Chiong W. Equipoise and the dilemma of randomized clinical trials. N Engl J Med 2011;364:2077.

6 Freedman B. Equipoise and the ethics of clinical research. N Eng/ J Med 1987;317:141-5

7 Miller F, Joffe S. Equipoise and the dilemma of randomized clinical trials. N Engl J Med 2011;364:476-80.

$8 \mathrm{Mann} \mathrm{H}$. Equipoise and the dilemma of randomized clinical trials. N Engl J Med 2011;364:2077.

9 McPherson K, Wennberg J, Hovind O, Clifford P. Small-area variations in the use of common surgical procedures: an international comparison of New England, England, and Norway. N Engl J Med 1982;307:1310-4.

10 Wennberg J, Gittelsohn A. Variations in medical care among small areas. Sci Am 1982;246:120-34

11 Petticrew M. Public health evaluation: epistemological challenges to evidence production and use. Evidence and Policy (forthcoming).

12 Thomson H, Atkinson R, Petticrew M, Kearns A. Do urban regeneration programmes improve public health and reduce health inequalities? A synthesis of the evidence from UK policy and practice (1980-2004). J Epidemiol Community Health 2006:60:108-15.

13 Han C JJ, Hoskins B, Green A. Perceptions of inequalities: implications for social cohesion. LLAKES research paper 35. 2012. www.llakes.org/wp-content/uploads/2012/07/35.-HanJanmaat-Hoskins-Green.pdf.

14 Gollust S, Lantz P, Ubel P. The polarizing effect of news media messages about the social determinants of health. Am J Publ Health 2009:99:2160-7.

15 Taber C, Lodge M. Motivated skepticism in the evaluation of political beliefs. Am J Polit Sci 2006;50:755-69.

16 Petticrew M. Plural evidence in public health evaluation: epistemological challenges to evidence production and use. Evidence Policy 2013;9:87-95.

17 Thomson H, Hoskins R, Petticrew M, Ogilvie D, Craig N, Quinn T, et al. Evaluating the health effects of social interventions. BMJ 2004;328:282-5.

18 Edwards P, Durand MA, Hollister M, Green J, Lutchmun S, Kessel A, et al. Scald risk in social housing can be reduced through thermostatic control system without increasing Legionella risk: a cluster randomised trial. Arch Dis Child 2011;96:1097-102.

19 Campbell D. Reforms as experiments. Am Psychologist 1969;24:409-29.

20 Grundy C, Steinbach R, Edwards P, Green J, Armstrong B, Wilkinson P. Effect of $20 \mathrm{mph}$ traffic speed zones on road injuries in London, 1986-2006: controlled interrupted time series analysis. BMJ 2009;339:b4469.

21 Shemilt I, Harvey I, Shepstone L, Swift L, Reading R, Mugford M, et al. A national evaluation of school breakfast clubs: evidence from a cluster randomized controlled trial and an observational analysis. Child Care Health Dev 2004;30:413-27.

22 Herring R, Thom B, Foster J, Franey C, Salazar C. Local responses to the Alcohol Licensing Act 2003: the case of Greater London. Drugs Educ Prevent Policy 2008:15:251-65

23 Dearlove J. The politics of policy in local government. Cambridge University Press, 1973.

24 Vize R. Councils have opportunity to show effectiveness in public health. Guardian 2013 Apr 5. www.guardian.co.uk/local-government-network/2013/apr/05/councils-opportunitypublic-health-transfer-nhs.

Accepted: 1 May 2013

Cite this as: BMJ 2013;346:f4016

(c) BMJ Publishing Group Ltd 2013 